\title{
Tanshinone IIA promotes non-amyloidogenic processing of amyloid precursor protein in platelets via estrogen receptor signaling to phosphatidylinositol 3-kinase/Akt
}

\author{
CHUN SHI ${ }^{1}$, XIAOMING ZHU ${ }^{2}$, JISHENG WANG ${ }^{1}$ and DAHONG LONG ${ }^{1}$ \\ ${ }^{1}$ Department of Anatomy, Guangzhou Medical University, Guangzhou, Guangdong, P.R. China; \\ ${ }^{2}$ Department of Imaging and Interventional Radiology, Prince of Wales Hospital, \\ The Chinese University of Hong Kong, Shatin, NT, Hong Kong
}

Received February 6, 2014; Accepted March 19, 2014

DOI: $10.3892 /$ br.2014.263

\begin{abstract}
Amyloid $\beta$ peptide $(\mathrm{A} \beta)$ is a proteolytic product of amyloid precursor protein (APP). Recent findings suggested that platelet-derived $A \beta$ is closely associated with the pathogenesis of atherosclerosis (AS). Tanshinone IIA (Tan IIA), a pharmacologically active component of the Chinese herb Salvia miltiorrhiza Bunge, has long been used to treat AS and was also identified as a phytoestrogen. However, it has not been elucidated whether Tan IIA intervenes with platelet APP processing and whether such an intervention is associated with its estrogenic activity. Using human platelets, this study demonstrated that Tan IIA promoted the non-amyloidogenic cleavage of APP via estrogenic activity. The phosphatidylinositol 3-kinase/Akt pathway may be involved in this effect of Tan IIA on platelet APP metabolism as a downstream effector of estrogen receptor signaling. This study aimed to extend the existing data and provide new insights into the mechanism underlying the vasoprotective effect of Tan IIA.
\end{abstract}

\section{Introduction}

$\mathrm{A} \beta$ is a peptide consisting of $36-43$ amino acids and is processed from the amyloid precursor protein (APP). APP is commonly processed by two competing pathways; one pathway involves cleavage by $\alpha$-secretase and then by $\gamma$-secretase, whereas the other involves processing by $\beta$-secretase and then by $\gamma$-secretase. The former pathway produces sAPP $\alpha$ and $\mathrm{p} 3$ and the latter pathway produces $\mathrm{APP} \beta$ and $\mathrm{A} \beta(1,2)$. It was previously demonstrated that the two pathways exert antagonistic effects on the generation of $A \beta$ (3). $\beta$-secretase cleavage of

Correspondence to: Professor Chun Shi, Department of Anatomy, Guangzhou Medical University, 195 Dongfeng Xi Road, Guangzhou 510182, P.R. China

E-mail: sallyshi431@126.com

Key words: tanshinone IIA, amyloid $\beta$ peptide, platelets, atherosclerosis, estrogen receptor
APP produces A $\beta$, whereas $\alpha$-secretase cleavage of APP within the $A \beta$ domain precludes the generation of $A \beta$ (3). Although best known as a component of senile plaques in the brain of patients with Alzheimer's disease, significant amounts of $A \beta$ are produced in the periphery (4). It was recently reported that the presence of $A \beta$ in the blood is intimately involved in the inflammatory pathology of atherosclerosis (AS) $(5,6)$.

It was demonstrated that $\mathrm{A} \beta$ peptides present in the atherosclerotic lesions are mainly derived from activated platelets (5). Platelets contain abundant APP and proteases involved in APP processing ( $\alpha-, \beta$ - and $\gamma$-secretases) (4). Platelet APP and APP proteolytic products (sAPP $\alpha, \operatorname{sAPP} \beta$ and A $\beta$ ) are stored in $\alpha$-granules and are released by agents that induce platelet degranulation, such as collagen (4). Platelets produce up to $90 \%$ of the $A \beta$ in circulation, mainly the $A \beta 40$ peptide (4). Therefore, platelet-derived $\mathrm{A} \beta$ may serve as a target for anti-AS therapy.

Tanshinone IIA (Tan IIA) is a pharmacologically active component isolated from the rhizome of the Chinese herb Salvia miltiorrhiza Bunge (Danshen) (7-9). Since Tan IIA has a quinoid structure and is easy to be oxidized and reduced, it may participate in various biochemical reactions in the human body. Emerging experimental studies and clinical trials demonstrated that Tan IIA is able to prevent atherogenesis, possibly through its antioxidant and anti-inflammatory actions (7-9). However, the precise mechanisms underlying the vasoprotective effect of Tan IIA have not been clearly determined. Tan IIA was recently identified as a new member of the phytoestrogen family $(7,10)$. Tan IIA at $5 \mu \mathrm{M}$ was found to increase estrogen response element-luciferase activity in an estrogen receptor (ER)-dependent manner in HeLa cells (7). The non-genomic action of ER is involved in a number of the anti-inflammatory actions of Tan IIA $(7,10)$. For example, in lipopolysaccharide-treated RAW 264.7 cells, Tan IIA was shown to inhibit the expression of inducible nitric oxide synthase, the production of nitric oxide and the release of inflammatory cytokines, such as interleukin (IL)-1 $\beta$, IL-6 and tumor necrosis factor- $\alpha$ via an ER-dependent pathway (10). In our previous studies, we demonstrated that ER-mediated PI3K/Akt signaling promotes $\alpha$-secretase cleavage of APP and inhibits the production of $A \beta(1,11)$. In this study, we aimed to 
assess whether Tan IIA modulates platelet APP metabolism via an ER-dependent pathway.

\section{Materials and methods}

Reagents. Tan IIA was purchased from Sigma (St. Louis, MO, USA). Phosphospecific antibodies to p-Akt (Ser473 and Thr308) and total Akt were obtained from Santa Cruz Biotechnology (CA, USA). The goat anti-rabbit IgG-horseradish peroxidase (HRP) was purchased from Invitrogen Life Technologies (Grand Island, NY, USA). Unless stated otherwise, all the chemicals were purchased from Sigma (St. Louis, MO, USA). Stock solutions $(1,000 \mathrm{X})$ of Tan IIA, the ER antagonist ICI182.780, the ER $\alpha$-specific antagonist methyl-piperidinopyrazole (MPP) and the phosphatidylinositol 3-kinase (PI3K) inhibitor LY294002 were prepared in ethanol and added to the platelet suspension at the indicated concentrations. Stock solutions of collagen ( $5 \mathrm{mg} / \mathrm{ml}$, human placental collagen type I) were prepared in water (the $\mathrm{pH}$ was adjusted to 3.0 with acetic acid). In all the experiments, an equivalent volume of ethanol $(0.1 \%)$ was added to the control group.

Subjects. A total of 12 healthy subjects, including 6 men and 6 women of mean age \pm SD 57.7 \pm 4.6 years (range $50-65$ years), were recruited in this study, all without complaints of cognitive or memory deficits. Major systemic, psychiatric or neurological conditions, hypertension, diabetes mellitus, tumors, autoimmune diseases, hypercholesterolaemia, drug or alcohol abuse and intake of anticoagulants were precluded for all the subjects. Written informed consent was obtained from all the participants prior to study initiation. This study was approved by the Institutional Board of Guangzhou Medical University (Guangzhou, China).

Platelet preparation and pharmacological treatments. Peripheral venous blood $(10 \mathrm{ml})$ was collected from each subject. The anticoagulated blood was immediately centrifuged at $150 \mathrm{xg}$ for $10 \mathrm{~min}$ at room temperature and the supernatant was the platelet-rich plasma (PRP) (1). The platelet counts of PRP were adjusted to $1 \times 10^{8} / \mathrm{ml}$ by platelet-free plasma obtained by centrifugation of the blood samples at 4,000 x $\mathrm{g}$ for $20 \mathrm{~min}$. To determine the effect of Tan IIA on platelet APP metabolism, $5 \mu \mathrm{M}$ Tan IIA was added to PRP, followed by incubation with gentle agitation at $37^{\circ} \mathrm{C}$ for $24 \mathrm{~h}$.

To determine whether Tan IIA affects platelet APP metabolism via ER-mediated cell signaling, the platelets were incubated with Tan IIA $(5 \mu \mathrm{M})$ in the presence or absence of ICI182.780 $(1 \mu \mathrm{M})$, MPP $(1 \mu \mathrm{M})$ or LY294002 $(10 \mu \mathrm{M})$ at $37^{\circ} \mathrm{C}$. Subsequently, the platelets were collected and resuspended in modified Tyrode's buffer $(150 \mathrm{mM} \mathrm{NaCl}$, 5 mM N-2-hydroxyethylpiperazine-N'-2-ethanesulfonic acid, $0.55 \mathrm{mM} \mathrm{NaH} \mathrm{PO}_{4}, 7 \mathrm{mM} \mathrm{NaHCO}$, $2.7 \mathrm{mM} \mathrm{KCl}$, $0.5 \mathrm{mM} \mathrm{MgCl}_{2}$ and $5.6 \mathrm{mM}$ glucose). The platelet suspension was then incubated with gentle agitation at $37^{\circ} \mathrm{C}$ for another $1 \mathrm{~h}$, followed by measurement of $\mathrm{A} \beta$ and sAPP $\alpha$ released from inactivated and collagen $(10 \mu \mathrm{g} / \mathrm{ml})$-activated platelets (1).

$s A P P \alpha$ levels. Platelet secretion of sAPP $\alpha$ was measured using an sAPP $\alpha$ ELISA kit (IBL International, Hamburg, Germany).
Following platelet treatment as described above, the medium was collected and sAPP $\alpha$ levels were measured according to the manufacturer's protocol. Spectrophotometric data were collected using the Victor-2 Multilabel counter (Perkin Elmer/Wallace, Akron, OH, USA) at a wavelength of $450 \mathrm{~nm}$.

$A \beta$ levels. Platelet secretion of $\mathrm{A} \beta$ was measured using a human A $\beta 40$ ELISA kit (Invitrogen, Camarillo, CA, USA). Following platelet treatment as described above, the medium was collected and $A \beta$ levels were measured according to the manufacturer's protocol. Spectrophotometric data were collected using the Victor-2 Multilabel counter at a wavelength of $450 \mathrm{~nm}$.

$\alpha$-secretase activity. In this study, the $\alpha$-secretase activity in living platelets and platelet lysates was measured using an $\alpha$-secretase activity kit (R\&D Systems, Minneapolis, MN, USA). Following treatment, the platelets were collected by centrifugation of the platelet suspension at 1,400 x $\mathrm{g}$ for $15 \mathrm{~min}$. Alternatively, the platelets were lysed in the lysis buffer provided in the kit and then treated with Tan IIA or vehicle, as described above. The $\alpha$-secretase activity in living platelets and platelet lysates was then measured by fluorescent spectroscopy according to the manufacturer's protocol. The cleavage-dependent release of fluorescence signal was quantified using the Victor-2 Multilabel counter at an excitation wavelength of $340 \mathrm{~nm}$ and an emission wavelength of $495 \mathrm{~nm}$.

The protein concentration of the platelet lysates was determined with a detergent-compatible protein assay kit (Bio-Rad, Hercules, CA, USA). Spectrophotometric data were obtained using the Victor-2 Multilabel counter at a wavelength of $650 \mathrm{~nm}$. The enzyme activities were corrected for protein content.

PI3K activity. The PI3K activity was determined using a commercially available PI3K ELISA kit (Echelon Biosciences Inc., Salt Lake City, UT) according to the manufacturer's instructions. Briefly, following drug treatment, the platelets were collected and lysed in ice-cold lysis buffer $[137 \mathrm{mM} \mathrm{NaCl}$, $20 \mathrm{mM}$ Tris- $\mathrm{HCl}$ (pH 7.4), $1 \mathrm{mM} \mathrm{CaCl}_{2}, 1 \mathrm{mM} \mathrm{MgCl}$, $0.1 \mathrm{mM}$ sodium orthovanadate, $1 \% \mathrm{NP}-40$ and $1 \mathrm{mM}$ phenylmethylsulphonyl fluoride]. PI3K was then immunoprecipitated with anti-PI3K antibody and protein A-sepharose beads. The PI3K activity in the immunoprecipitates was then assayed by PI3K ELISA according to the manufacturer's instructions. The spectrophotometric data were obtained using the Victor-2 Multilabel counter at a wavelength of $450 \mathrm{~nm}$. The protein concentration in the platelet lysates was determined as described above. The activity of PI3K was corrected for protein content.

$p$-Akt levels. Following drug treatment, the platelets were collected by centrifugation of the platelet suspension at $1,400 \mathrm{x} g$ for $15 \mathrm{~min}$. The protein levels of $\mathrm{p}$-Akt were measured by western blot analysis. Briefly, after boiling in two-fold loading buffer [100 nM Tris- $\mathrm{HCl}(\mathrm{pH} 6.8)$ $4 \%$ sodium dodecyl sulfate, $200 \mathrm{nM}$ dithiothreitol, $0.2 \%$ bromophenol blue and $20 \%$ glycerol] for $5 \mathrm{~min}$, the proteins (20 $\mu \mathrm{g}$ per well) were subjected to $12 \%$ SDS-PAGE at $100 \mathrm{~V}$ for $3 \mathrm{~h}$ and then transferred onto nitrocellulose membranes using a semi-dry transfer apparatus (Bio-Rad). The transfer 

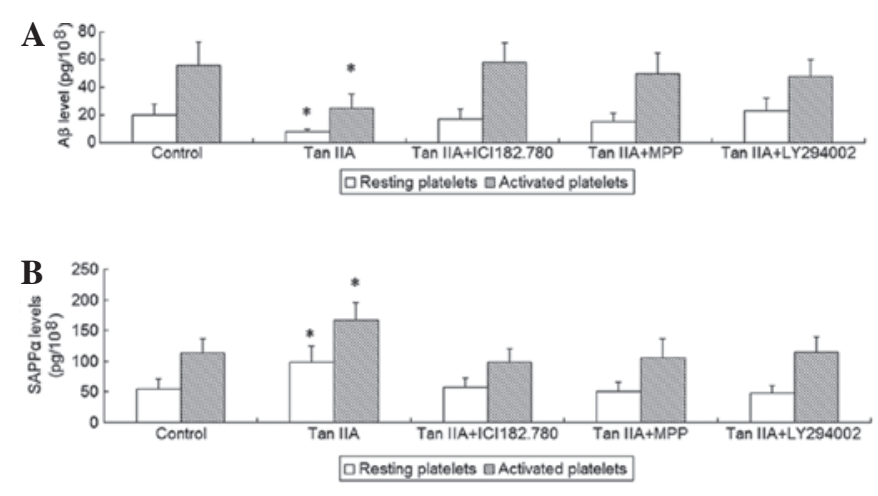

Figure 1. Estrogenic effect of Tanshinone IIA (Tan IIA) on platelet amyloid precursor protein (APP) processing. The platelets were treated with $5 \mu \mathrm{M}$ Tan IIA in the presence or absence of the estrogen receptor (ER) antagonist ICI182.780 $(1 \mu \mathrm{M})$ or the ER $\alpha$-specific antagonist methyl-piperidinopyrazole (MPP) $(1 \mu \mathrm{M})$ or the phosphatidylinositol 3-kinase (PI3K) inhibitor LY294002 $(10 \mu \mathrm{M})$ at $37^{\circ} \mathrm{C}$ for $24 \mathrm{~h}$. The (A) A $\beta$ and (B) sAPP $\alpha$ released into the medium were measured using the ELISA kits for $A \beta$ and sAPP $\alpha$, respectively. Values are presented as means \pm SD of five determinations. "P<0.01 vs. the control group (vehicle-only).

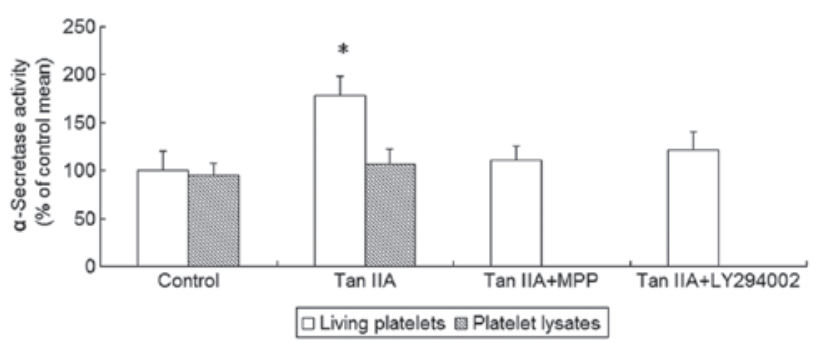

Figure 2. Estrogenic effect of Tanshinone IIA (Tan IIA) on platelet $\alpha$-secretase activity. The platelets were treated with Tan IIA in the presence or absence of the estrogen receptor $\alpha$-specific antagonist methyl-piperidinopyrazole (MPP) or the phosphatidylinositol 3-kinase (PI3K) inhibitor LY294002 at $37^{\circ} \mathrm{C}$ for $24 \mathrm{~h}$. Alternatively, the platelets were lysed and then incubated with Tan IIA at $37^{\circ} \mathrm{C}$ for $24 \mathrm{~h}$. Subsequently, $\alpha$-secretase activity in living platelets and the platelet lysates were measured using an $\alpha$-secretase activity kit. Values are presented as means \pm SD of five determinations. ${ }^{*} \mathrm{P}<0.01$ vs. the control group (vehicle-only).

of the proteins was performed for $1 \mathrm{~h}$ at $10 \mathrm{~V}$. The blots thus obtained were rinsed in PBST solution [100 mM phosphate buffer ( $\mathrm{pH} 7.5$ ) containing $150 \mathrm{mM} \mathrm{NaCl}$ and $0.05 \%$ Tween-20] and blocked with 5\% non-fat dry milk (Bio-Rad, Hercules, CA, USA) in PBST for $1 \mathrm{~h}$. The blots were then incubated with primary antibodies against p-Akt (Ser473 and Thr308) $(1: 1,000)$ and total Akt $(1: 1,000)$ on a platform shaker overnight at $4^{\circ} \mathrm{C}$. After rinsing with PBST, the blots were incubated with 1:5,000 goat anti-rabbit IgG-HRP for $2 \mathrm{~h}$ and then rinsed with PBST. The protein band was visualized using X-ray film and the band intensities were determined with the Bio-Rad GS800 densitometer equipped with 'Quantity One' software package.

Statistical analysis. The statistical analysis was performed by SPSS software, version 15.0 (SPSS Inc., Chicago, IL, USA). After the Ryan-Joiner test for normal distribution and the Levene's test of Equal Variances for variance homogeneity, data were analyzed by one-way analysis of variance followed by pairwise t-tests.

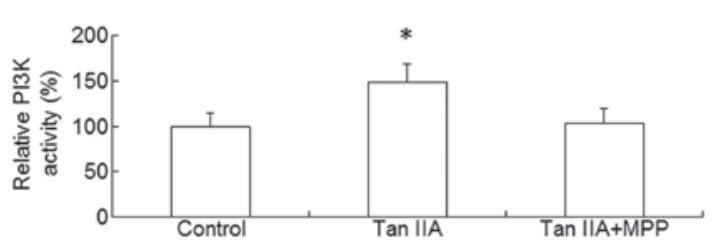

Figure 3. Estrogenic effect of Tanshinone IIA (Tan IIA) on platelet phosphatidylinositol 3-kinase (PI3K) activity. The platelets were treated with Tan IIA in the presence or absence of the estrogen receptor $\alpha$-specific antagonist methyl-piperidinopyrazole (MPP) at $37^{\circ} \mathrm{C}$ for $24 \mathrm{~h}$. Subsequently, the platelets were collected and lysed. PI3K activity was then measured using a PI3K ELISA kit. Values are presented as means \pm SD of five determinations. ${ }^{*} \mathrm{P}<0.01$ vs. the control group (vehicle-only).

$\mathbf{A}$

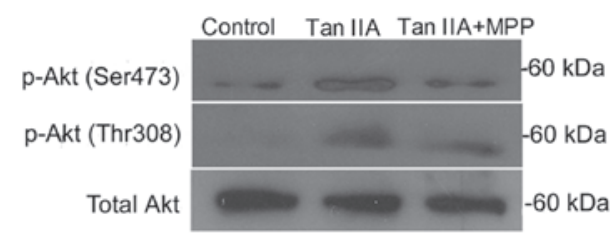

B

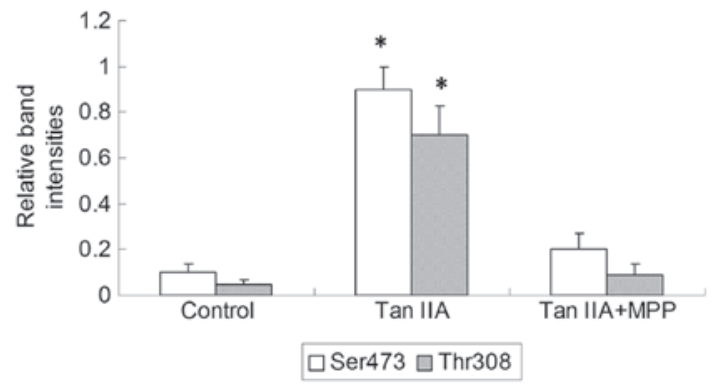

Figure 4. Estrogenic effect of Tanshinone IIA (Tan IIA) on platelet p-Akt levels. The platelets were treated with Tan IIA in the presence or absence of the estrogen receptor $\alpha$-specific antagonist methyl-piperidinopyrazole (MPP) at $37^{\circ} \mathrm{C}$ for $24 \mathrm{~h}$. Subsequently, the platelets were collected and lysed. The platelet lysates $(20 \mu \mathrm{g})$ were then analyzed for Akt activation by western blot analysis, using p-Akt (Ser473 and Thr308) antibodies. The same crude lysates $(20 \mu \mathrm{g})$ were also analyzed using an antibody that recognizes total Akt. (A) Representative western blots of platelet p-Akt; (B) The relative ratios of p-Akt to total Akt band density were presented as means \pm SD of four determinations in a bar chart. ${ }^{*} \mathrm{P}<0.01$ vs. the control group (vehicle-only).

\section{Results}

Tan IIA promotes non-amyloidogenic APP processing in human platelets. To investigate the effects of Tan IIA on platelet APP metabolism, the platelets were incubated with $5 \mu \mathrm{M}$ Tan IIA for $24 \mathrm{~h}$; subsequently, sAPP $\alpha$ and A $\beta$ released from resting and activated platelets were measured. It was observed that Tan IIA treatment increased the release of $\operatorname{sAP} \alpha$ and decreased the release of $A \beta$ from resting as well as from collagen-activated platelets $(\mathrm{P}<0.01$, Fig. 1$)$, suggesting that Tan IIA promotes non-amyloidogenic APP processing in platelets.

Non-amyloidogenic APP processing is mediated by $\alpha$-secretase. Therefore, we next investigated the effect of Tan IIA on $\alpha$-secretase activity and observed that Tan IIA enhanced $\alpha$-secretase activity in platelets $(P<0.01$, Fig. 2$)$. We hypothesized that the promotion of non-amyloidogenic APP processing by Tan IIA required structured cellular functions, such as signal transduction cascades, rather than direct activation of $\alpha$-secretase. To exclude the possibility of direct activation, the platelet lysates were treated with Tan IIA 
(5 $\mu \mathrm{M})$. However, there was no statistically significant effect of Tan IIA on $\alpha$-secretase activity in the platelet lysates $(\mathrm{P}>0.05$, Fig. 2), suggesting that $\alpha$-secretase activation by Tan IIA may not be mediated by a direct molecular interaction between $\alpha$-secretase and Tan IIA. These findings indicate the involvement of signal transduction pathways in Tan IIA-mediated $\alpha$-secretase activation.

ER-mediated PI3K/Akt signaling is involved in the effect of Tan IIA on platelet APP metabolism. It was demonstrated that ER-mediated PI3K/Akt signaling is involved in APP metabolism $(1,11)$. Tan IIA is known to be a phytoestrogen and may activate ER-mediated signaling, such as PI3K/Akt $(7,10)$. To determine whether Tan IIA modulates APP metabolism via ER signaling, the platelets were incubated with Tan IIA in the presence or absence of the ER antagonist ICI182.780 or the ER $\alpha$-specific antagonist MPP or the PI3K inhibitor LY294002; subsequently, sAPP $\alpha$ and $\mathrm{A} \beta$ released from resting and activated platelets as well as the activity of $\alpha$-secretase were measured. It was observed that the two ER antagonists and the PI3K inhibitor were able to abrogate the effects of Tan IIA on APP metabolism and $\alpha$-secretase acitivity $(\mathrm{P}<0.01$, Figs. 1 and 2). To determine whether the PI3K/Akt pathway is a downstream effector of activated ER, the platelets were treated with Tan IIA in the presence or absence of the ER antagonist MPP; subsequently, the PI3K activity and p-Akt levels were measured. It was observed that Tan IIA upregulated the PI3K activity and p-Akt levels and this effect was suppressed in the presence of MPP ( $\mathrm{P}<0.01$, Figs. 3 and 4). These results suggest that ER-mediated PI3K/Akt signaling may be involved in the effect of Tan IIA on APP metabolism.

\section{Discussion}

It was demonstrated that the aberrant deposition of $A \beta$, predominantly $A \beta 40$, in the intima is pathologically significant in the development of $\mathrm{AS}(5,6)$. The activated platelets were reported to be the principal source of $A \beta$ in AS lesions (5). Therefore, platelet APP metabolism may serve as a target for AS therapy. Tan IIA, the major active ingredient responsible for the beneficial actions of Danshen, has long been used for the prevention and treatment of AS (7-9) and was recently identified as a phytoestrogen $(7,10)$. However, it has not been elucidated whether Tan IIA intervenes in platelet APP processing and whether such an intervention is associated with its estrogenic activity.

In this study, we demonstrated that Tan IIA promoted the release of sAPP $\alpha$ and inhibited the secretion of A $\beta$ from resting as well as from activated platelets, suggesting that Tan IIA enhances non-amyloidogenic APP processing in platelets. Additionally, the promotion of non-amyloidogenic APP processing by Tan IIA required activation of PI3K/Akt signaling rather than direct interaction between $\alpha$-secretase and Tan IIA. In our previous studies, we reported that ER-mediated PI3K/Akt signaling is involved in APP metabolism $(1,11)$, suggesting that the effect of Tan IIA on APP metabolism may be associated with its estrogenic activity. In this study, the effect of Tan IIA on platelet APP metabolism was inhibited blocked by the ER antagonists and the PI3K inhibitor. In addition, Tan IIA upregulated PI3K activity and
p-Akt levels, an effect that was suppressed by the ER antagonists. These data suggest that Tan IIA modulates platelet APP metabolism, possibly through ER signaling to PI3K/Akt. In accordance with this finding, our previous studies demonstrated that treatment of HT22 cells, SH-SY5Y cells stably expressing the Swedish mutant APP and ovariectomized rats with phytoestrogens, such as ginsenoside Rg1 and bilobalide, increases the secretion of $\operatorname{sAPP} \alpha$, enhances $\alpha$-secretase activity and decreases the production of $\mathrm{A} \beta$ via ER-mediated PI3K/Akt signaling (11-13).

It is known that ER regulates cellular function through at least two signaling pathways, including the nuclearER-mediated genomic pathway and the membrane ER-mediated nongenomic pathway (14). In platelets, Tan IIA modulates APP metabolism possibly via membrane ER-mediated nongenomic signaling. It was demonstrated that membrane ERs reside in the lipid rafts $(15,16)$, where the ER isoform, ER $\alpha$, activates PI3K through membrane recruitment and direct interaction with PI3K (17). ER $\alpha$ binds in a ligand-dependent manner to the $\mathrm{p} 85 \alpha$ regulatory subunit of $\mathrm{PI} 3 \mathrm{~K}$, leading to the phosphorylation of Akt (18).

Given that blood $A \beta$ is intimately associated with the inflammatory pathology of AS, the finding that Tan IIA promotes non-amyloidogenic APP processing in platelets sheds new light on the mechanism underlying the vasoprotective effect of Tan IIA. Of note, blood sAPP $\alpha$ and A $\beta$ were demonstrated to exert antagonistic effects on platelet function (4). A $\beta$ augments physiological agonist-induced platelet activation and platelet adhesion, whereas $\mathrm{SAPP} \alpha$ inhibits platelet aggregation and degranulation (4). Therefore, there may be an enhanced interaction between the released sAPP $\alpha$ and Tan IIA in the mechanism of Tan IIA-induced reduction in platelet $A \beta$ release: Tan IIA increases the release of sAPP $\alpha$ from platelets, possibly leading to a sustained decrease of platelet $\mathrm{A} \beta$ release via inhibition of platelet degranulation.

In conclusion, this study demonstrated, using human platelets, that Tan IIA promotes the non-amyloidogenic pathway of APP cleavage via its estrogenic activity. The PI3K/Akt pathway may be involved in the effect of Tan IIA on APP metabolism as a downstream effector of ER signaling. Since the APP proteolytic product, $A \beta$, is involved in the inflammatory pathology of AS, these findings may contribute to a better understanding of the vasoprotective effect of Tan IIA.

\section{Acknowledgements}

The authors are grateful for the support from the National Natural Science Foundation of China (grant nos. 81370395 and 81200846) and the Guangdong Natural Science Foundation (grant no. S2012040007858 and S2013010014468).

\section{References}

1. Shi C, Na N, Zhu X and Xu J: Estrogenic effect of ginsenoside $\mathrm{Rg} 1$ on APP processing in post-menopausal platelets. Platelets 24: 51-62, 2013.

2. Gandy S and DeKosky ST: Toward the treatment and prevention of Alzheimer's disease: rational strategies and recent progress. Annu Rev Med 64: 367-383, 2013.

3. Mandel S, Amit T, Reznichenko L, Weinreb O and Youdim MB: Green tea catechins as brain-permeable, natural iron chelators-antioxidants for the treatment of neurodegenerative disorders. Mol Nutr Food Res 50: 229-234, 2006. 
4. Li QX, Fuller SJ, Beyreuther K and Masters CL: The amyloid precursor protein of Alzheimer disease in human brain and blood. J Leukoc Biol 66: 567-574, 1999.

5. Kokjohn TA, Van Vickle GD, Maarouf CL, et al: Chemical characterization of pro-inflammatory amyloid-beta peptides in human atherosclerotic lesions and platelets. Biochim Biophys Acta 1812: 1508-1514, 2011.

6. Howlett GJ and Moore KJ: Untangling the role of amyloid in atherosclerosis. Curr Opin Lipidol 17: 541-547, 2006.

7. Fan GW, Gao XM, Wang H, et al: The anti-inflammatory activities of tanshinone IIA, an active component of TCM, are mediated by estrogen receptor activation and inhibition of iNOS. J Steroid Biochem Mol Biol 113: 275-280, 2009.

8. Xu S, Little PJ, Lan T, et al: Tanshinone II-A attenuates and stabilizes atherosclerotic plaques in apolipoprotein-E knockout mice fed a high cholesterol diet. Arch Biochem Biophys 515: 72-79, 2011.

9. Gao S,Liu Z,Li H,Little PJ,Liu P and Xu S: Cardiovascular actions and therapeutic potential of tanshinone IIA. Atherosclerosis 220 : 3-10, 2012 .

10. Fan G, Zhu Y, Guo H, Wang X, Wang H and Gao X: Direct vasorelaxation by a novel phytoestrogen tanshinone IIA is mediated by nongenomic action of estrogen receptor through endothelial nitric oxide synthase activation and calcium mobilization. J Cardiovasc Pharmacol 57: 340-347, 2011.

11. Shi C, Zheng DD, Fang L, Wu F, Kwong WH and $\mathrm{Xu} \mathrm{J}$ : Ginsenoside Rg1 promotes nonamyloidgenic cleavage of APP via estrogen receptor signaling to MAPK/ERK and PI3K/Akt. Biochim Biophys Acta 1820: 453-460, 2012.
12. Shi $\mathrm{C}, \mathrm{Wu} \mathrm{F}, \mathrm{Xu} \mathrm{J}$ and Zou J: Bilobalide regulates soluble amyloid precursor protein release via phosphatidyl inositol 3 kinase-dependent pathway. Neurochem Int 59: 59-64, 2011.

13. Shi C, Zheng DD, Wu FM, Liu J and Xu J: The phosphatidyl inositol 3 kinase-glycogen synthase kinase $3 \beta$ pathway mediates bilobalide-induced reduction in amyloid $\beta$-peptide. Neurochem Res 37: 298-306, 2012.

14. Grove-Strawser D, Boulware MI and Mermelstein PG: Membrane estrogen receptors activate the metabotropic glutamate receptors mGluR5 and mGluR3 to bidirectionally regulate CREB phosphorylation in female rat striatal neurons. Neurosci 170: 1045-1055, 2010.

15. Gilad LA and Schwartz B: Association of estrogen receptor beta with plasma-membrane caveola components: implication in control of vitamin D receptor. J Mol Endocrinol 38: 603-618, 2007.

16. Chambliss KL, Yuhanna IS, Mineo C, et al: Estrogen receptor alpha and endothelial nitric oxide synthase are organized into a functional signaling module in caveolae. Circ Res 87: E44-E52, 2000.

17. Losel RM, Falkenstein E, Feuring M, Schultz A, Tillmann HC, Rossol-Haseroth $\mathrm{K}$ and Wehling M: Nongenomic steroid action: controversies, questions, and answers. Physiol Rev 83: 965-1016, 2003.

18. Simoncini T, Hafezi-Moghadam A, Brazil DP, Ley K, Chin WW and Liao JK: Interaction of oestrogen receptor with the regulatory subunit of phosphatidylinositol-3-OH kinase. Nature 407: $538-541,2000$ 